\title{
Update on rescue therapies in patients with lamivudine-resistant chronic hepatitis B
}

This article was published in the following Dove Press journal:

Drug Design, Development and Therapy

19 August 2013

Number of times this article has been viewed

\section{Daniel C Chao \\ $\mathrm{Ke}-\mathrm{Q}$ in $\mathrm{Hu}$}

Division of Gastroenterology and Hepatology, University of California, Irvine Medical Center, Orange, CA, USA
Correspondence: Ke-Qin Hu

Division of Gastroenterology and Hepatology, University of California, Irvine, I0I The City Drive, Building 56, Rt 8I, Room 237,

Orange, CA 92868, USA

$\mathrm{Tel}+\mathrm{I} 7 \mathrm{l} 44566926$

Fax +I 7144563283

Email kqhu@uci.edu

\begin{abstract}
Chronic hepatitis B continues to be a global problem, with an estimated 240 million cases according to the World Health Organization. Chronic infection with the hepatitis $B$ virus (HBV) is associated with cirrhosis, hepatic decompensation, and hepatocellular carcinoma. There are currently several US Food and Drug Administration-approved medications for treating chronic hepatitis B, with Lamivudine (LAM) being the first oral agent made available. The major problem with LAM is significantly decreased effectiveness over time due to the development of anti-HBV resistance that can lead to virologic and biochemical breakthrough as well as hepatitis B flare, progression of liver disease, and decompensation of pre-existing cirrhosis. Despite its high anti-HBV resistant rate, LAM remains widely used in underdeveloped countries due to its wide availability and low cost compared to other antiviral medications, including those that are more effective. Therefore, it is still clinically important to learn how to prevent and treat LAM resistant strains of HBV. Several regimens with the other available antiviral agents have been studied, including switching to monotherapy with either Adefovir, Entecavir, or Tenofovir, adding Adefovir to LAM, and switching to a combination of Adefovir and Entecavir. This review article will examine molecular mechanisms and diagnosis of LAM anti-HBV resistance, risks for and approaches to reduce LAM anti-HBV resistance, and currently available rescue therapy regimens for LAM resistance.
\end{abstract}

Keywords: chronic hepatitis B, nucleot(s)ide analogs, anti-viral resistance, lamivudine, adefovir, entecavir, tenofovir

\section{Introduction}

In the United States, the incidence of chronic hepatitis $B$ virus infection (CHB), defined as a positive serum hepatitis B surface antigen (HBsAg) for more than 6 months, has declined by over $80 \%$ since the 1990 s, according to the US Centers for Disease Control and Prevention. This is primarily due to widespread vaccination against hepatitis $B$ virus (HBV). ${ }^{1}$ Since the advent of the HBV vaccine in 1981, 179 countries have implemented routine HBV vaccination to all newborns, including the United States. Despite the declining incidence, there were an estimated 240 million cases of chronic hepatitis B globally in July 2012, according to the World Health Organization. ${ }^{2}$ The American Association for the Study of Liver Diseases (AASLD) estimated in 2009 that of the 350 million chronically infected individuals worldwide, 1.25 million are located in the United States. ${ }^{3}$ Therefore, there is still a significant global healthcare burden from CHB.

Morbidity and mortality from CHB is due to the development of cirrhosis, hepatic decompensation, and hepatocellular carcinoma (HCC). While the cause of 
HCC varies geographically, as many as $20 \%$ of cases in the United States and up to $60 \%$ of cases in areas with high HBV prevalence (eg, Asia and Africa) are HBV-related. ${ }^{4}$ Approximately 600,000 deaths are attributed to hepatitis B globally each year.

The first therapeutic agent for hepatitis B, conventional interferon alfa, was administered subcutaneously. Development of the pegylated form, which had significantly better response rates, led to loss of hepatitis $\mathrm{B}$ e antigen (HBeAg) in $33 \%$ of treated patients, compared to $12 \%$ in untreated controls. Only $25 \%$ of those treated achieved an undetectable HBV DNA level. There was particularly poor response among immune tolerant individuals. ${ }^{5,6}$

Lamivudine (LAM) was the first approved oral nucleoside analog (NA) agent for the treatment of CHB. The first doubleblinded, randomized controlled trial demonstrated that LAM $100 \mathrm{mg}$ daily for one year led to $\mathrm{HBeAg}$ seroconversion to hepatitis $\mathrm{B}$ e antibody ( $\mathrm{HBe} \mathrm{Ab})$, and an undetectable serum HBV DNA in $16 \%$ of patients. ${ }^{7}$ LAM was well tolerated, with no serious adverse effects. This, and other positive studies, led to widespread use of LAM to treat CHB from 1998 to 2004 . $^{6,7}$

The advantages of LAM include a lack of significant adverse effects, proven efficacy in reducing viral load, decreasing severity of hepatitis, and inducing $\mathrm{HBeAg}$ seroconversion, and relatively low cost. LAM is also useful in treating infection with human immunodeficiency virus (HIV), and is included in some regimens for HBV and HIV co-infection.

One disadvantage of LAM is less efficacy compared to newer oral antiviral medications such as Entecavir (ETV) and Tenofovir (TDF) in suppressing HBV DNA replication. ${ }^{8}$ The major disadvantages of LAM are the risk of resistance, and a recurrence rate if treatment is discontinued. In a review of 998 patients on long-term LAM monotherapy for CHB, acute hepatitis flares and worsening liver disease with hepatic decompensation and death were among the serious adverse effects felt to be related to the development of resistance. ${ }^{9}$ The need for rescue therapy in the setting of resistance related virological breakthrough or acute exacerbation of disease also places patients at risk for developing multidrug resistance, which will be discussed later.

In 2005 and beyond, Adefovir (ADV), ETV, Telbivudine (LdT), and TDF have also been approved as more effective alternative NA treatment agents for CHB. Currently, ETV and TDF are the preferred medications for CHB in developed countries and regions due to their improved efficacy and superior resistance profile, with multiple studies showing that long-term and effective HBV treatment leads to both histological and biochemical improvement in patients with CHB. ${ }^{10,11}$

Since LAM remains widely used as primary therapy for $\mathrm{CHB}$ in areas where access to more expensive, more efficacious HBV medications is limited, it is important to know the available rescue therapies in patients with lamivudine-resistant CHB.

\section{Diagnosis of LAM anti-HBV resistance Molecular mechanisms for LAM anti-HBV resistance}

The high replication rate of $\mathrm{HBV}$, coupled with a lack of effective proofreading in the HBV polymerase, is the basis for development of mutations in the viral genome. Mutations that alter the binding sites of antiviral medications to HBV are the molecular mechanisms for developing drug resistance.

Table 1 summarizes DNA sequences in the HBV genome that are known to cause resistance to specific antiviral medications. Resistance to LAM during HBV treatment develops from mutations within the tyrosine-methionineaspartate-aspartate (YMDD) motif of the $\mathrm{C}$ domain and $\mathrm{B}$ domain of the HBV genome., ${ }^{72}$ The development of LAM resistant $\mathrm{HBV}$ strains leads to a decrease in response to LAM treatment as high as 100 -fold with subsequent loss of virologic, biochemical, and histologic benefits. In these cases, the incidence of acute exacerbation of hepatitis B, and the rate of progression to cirrhosis and hepatocellular carcinoma (HCC) are increased. ${ }^{13}$

Certain genetic mutations confer cross-resistance to multiple drugs, which is important in the choice of alternative therapies in the setting of LAM resistance. This will be discussed further in the therapy section.

The genetic barrier to resistance is defined as the number and type of mutations required to confer drug resistance. Both transitions and transversions within the HBV genome

Table I Mutations associated with resistance to antiviral medications

\begin{tabular}{|c|c|}
\hline Drug name & Known mutations \\
\hline Lamivudine & rtM204V/I/S, rtL80V, rtVI74 L, rtLI80M \\
\hline Telbivudine & $\mathrm{rtM} 204 \mathrm{l}, \mathrm{rtL} 229 \mathrm{~W} / \mathrm{V}$ \\
\hline Entecavir & $\begin{array}{l}r t M 204 \mathrm{~V} / \mathrm{I}+\mathrm{rtLI} 180 \mathrm{M} \pm \mathrm{rtL} 169 \mathrm{~T} \pm \mathrm{rtSI} 184 \mathrm{G} \pm \\
\mathrm{rtS} 202 \mathrm{G} / \mathrm{I} \pm \mathrm{rtM} 250 \mathrm{r} ; \mathrm{rtT} 184 \mathrm{~A} / \mathrm{G} / / \mathrm{S}\end{array}$ \\
\hline Lamivudine and entecavir & VI73L, LI80M, M204I, M204V \\
\hline Adefovir & rtAI8IT/V, rtN236T, rt|244V \\
\hline Tenofovir & None confirmed \\
\hline
\end{tabular}

Note: data acquired from references ${ }^{12,60-63}$. 
can occur, with different degrees of clinical consequence. ${ }^{14}$ Therefore, anti-HBV medications with a low barrier to resistance (eg, LAM, LdT) have a higher rate of resistance over time compared to those with a high barrier to resistance (eg, ETV, TDF). Given that higher rates of viral replication also leads to increased probability of mutations leading to drug resistance, medications with lower potency for viral suppression (eg, ADV) will have a higher risk of resistance compared to those with higher potency (eg, ETV, TDF). ${ }^{14}$

\section{Laboratory testing for LAM anti-HBV resistance}

There are many types of diagnostic tests available for identifying LAM resistant strains of HBV, with varying sensitivities. Polymerase chain reaction (PCR)-based sequencing of the viral genome, which is the most common method used in clinical trials, can detect mutants that are present in at least $20 \%$ of the total viral population. For known mutation sequences, restriction fragment length polymorphisms (RFLP) and reverse hybridization line-probe assays can detect polymorphisms that are only present in 5\% of the total viral population, and ultra-deep pyrosequencing can be up to 50 times more sensitive than RFLP. ${ }^{15,16}$

\section{Timing and pattern of LAM anti-HBV resistance}

The initial clinical manifestation of LAM resistance is virological breakthrough (VBT), also known as secondary treatment failure, defined as an increase in HBV DNA of more than $1 \log _{10} \mathrm{IU} / \mathrm{mL}$ from the lowest detectable level achieved by a patient, or redetection of HBV DNA at levels $>10$-fold of the lower limit of detection of the HBV DNA assay after having an undetectable result. The mutations in the virus genotype will have already occurred at that point. In analysis of resistance trials, the incidences of genotypic resistance patterns and VBT were different. The reason for the difference was felt to be from VBT related to medication non-compliance, with as high as $30 \%$ of VBT attributed to non-compliance in clinical trials. Other manifestations of resistance, which follow VBT, include biochemical breakthrough with elevation of alanine aminotransferase (ALT) levels above normal after previously achieving normalization, acute hepatitis flare defined as elevation of ALT to levels greater than three times the upper limit of normal, decompensation of existing cirrhosis, and fulminant hepatic failure. The severity of symptoms is higher in those with advanced baseline liver disease. . $^{3,17}$

In a study on long-term LAM treatment for HBeAgpositive CHB (159 of whom completed the full 5 years of therapy, and 592 completed 4 years), the proportion of patients who developed hepatitis flares increased from year 1 of therapy to year 2, but remained stable from years 2 through 5 (10\% versus $18 \%-21 \%)$. Among these patients, the percentage with development of LAM resistance within 1-year of the hepatitis flare, and therefore felt to have a resistance-associated flare, was $43 \%$ during the first year of therapy, rose to $67 \%$ during year 2 of therapy, then rose further to a mean of $87 \%$ during years 3 through 5 . The proportion of patients with biochemical changes, defined as increase in serum bilirubin levels and/or increase in prothrombin time from baseline or international normalized ratio greater than 1.5 , did not increase with years of resistance. Unfortunately, this study did not evaluate HBV DNA levels. ${ }^{9}$

Given these findings, monitoring the HBV DNA and ALT at regular intervals, even as frequently as once every 3 months, may be necessary to diagnose LAM resistance.

\section{Risk factors for LAM anti-HBV resistance}

There are many clinical and environmental factors that lead to increased risk of LAM resistance. Understanding these can help reduce LAM anti-HBV resistance in patients that need antiviral treatment for CHB.

\section{Treatment duration}

In the trial study by Lai et al, $14 \%$ of patients exhibited the YMDD mutation, which was not present in any of the patients in the placebo group. ${ }^{7}$ Another review of 998 patients revealed $23 \%$ resistance at 1 -year, and $65 \%$ resistance at 5 years. ${ }^{9}$ Resistance at 5 -year follow up has been as high as $80 \%$, and multiple studies have demonstrated this trend of increasing incidence of resistance with longer duration of treatment.

\section{Suboptimal response to anti-viral therapy}

One of the core concepts is that active virus replication is required to develop resistant strains. ${ }^{14,18}$ Primary non-response to treatment, defined as a $<1 \log _{10}$ drop in HBV DNA at week 12, as well as partial response (still detectable HBV DNA levels after 24 weeks of treatment) are both risk factors in developing anti-HBV resistance. ${ }^{14}$ In a study of 150 Asian $\mathrm{HBeAg}$-positive patients during a 30-month treatment period with LAM, the incidence of resistance was directly related to the week $24 \mathrm{HBV}$ DNA levels (8\%, 13\%, 32\%, and $63 \%$ resistance for $\mathrm{HBV} \mathrm{DNA}<40 \mathrm{IU} / \mathrm{mL},<200 \mathrm{IU} / \mathrm{mL}$, $<2000 \mathrm{IU} / \mathrm{mL}$, and $>2000 \mathrm{IU} / \mathrm{mL}$, respectively). ${ }^{15}$ These results were further reflected in trials comparing different 
HBV treatments, such as the LdT versus LAM comparison study. One year LAM resistance rates were 3\%, 10\%, $15 \%$, and $17 \%$ in $\mathrm{HBeAg}$ positive patients and $2 \%, 20 \%$, $38 \%$, and $50 \%$ in $\mathrm{HBeAg}$ negative patients with $\mathrm{HBV}$ DNA levels $\leq 60,60-199,200-1999$, and $\geq 2000 \mathrm{IU} / \mathrm{mL}$ at week 24, respectively. ${ }^{19}$ In the GLOBE study comparing LdT to LAM for treatment of CHB, HBV DNA levels after 24 weeks of treatment were found to be the greatest predictor of long-term efficacy, confirmed by later studies comparing other predictors such as ALT response, baseline ALT, and baseline HBV DNA level. ${ }^{20}$

\section{High baseline HBV DNA load}

Although a literature review found no direct studies comparing response to treatment and development of resistance based on HBV viral load, it seems intuitive that a higher viral load leads to a higher rate of resistance, based on the higher likelihood of suboptimal response to treatment.

\section{Loss of access to medications}

Given that $\mathrm{CHB}$ has a high prevalence in many parts of the world with limited access to medical care, the fact that antiviral therapy for $\mathrm{CHB}$ needs to be continued for years is a considerable obstacle to treatment adherence. Inability to afford long-term therapy, inability for pharmacies to maintain a supply of medication, or lack of access to clinical follow up can all contribute to development of resistance. In the former, partial response and premature cessation of treatment was previously discussed as a risk factor for resistance. For the latter, those who had a lack of response or partial response to treatment would not be detected and would actually develop resistance through prolonged compliance with therapy.

\section{Non-compliance with anti-viral therapy}

Since failing to achieve an undetectable HBV DNA level is a positive predictor for developing LAM resistance, noncompliance with anti-viral therapy increases the rate of resistance due to a behaviorally induced decrease in drug efficacy and reduction of viral suppression. ${ }^{12}$

\section{High resistance HBV genotypes}

There are eight known HBV genotypes (A through $\mathrm{H}$ ), with varying global distribution, and there is conflicting data on whether a particular genotype leads to increased risk of anti-HBV resistance. One study of 40 patients with genotypes $\mathrm{B}$ and $\mathrm{C}$ receiving LAM monotherapy showed a statistically significant difference in the rate of LAM resistance within the first 12 months (odds ratio for genotype B versus C 8.27, $P=0.004) .{ }^{21}$ However, a larger study involving 129 patients of genotypes $\mathrm{A}, \mathrm{D}$, and $\mathrm{F}$ showed no association between development of resistance and genotypes A and D (27/50 and $16 / 50$, respectively, $P=0.189)$, despite a higher incidence of resistance mutations among genotype A (59.1\%) compared to D (30.3\%). ${ }^{22}$ Therefore, additional studies may need to be done to formulate definitive conclusions.

\section{HIV co-infection}

The prevalence of HIV and HBV co-infection is $5 \%-10 \%$ in most regions of the world, including North America, Europe, Australia, and Brazil. LAM, ADF, and TDF have all been approved for the treatment of both HBV and HIV infection. The recommended dose of Lamivudine is $150 \mathrm{mg}$ twice daily, as opposed to $100 \mathrm{mg}$ daily for HBV infection alone. ${ }^{3}$ Long-term studies have shown significant rates of resistance, up to $20 \%$ of patients per year. This was based on 2-year follow-up of 66 patients treated with LAM monotherapy. ${ }^{23}$ A second study retrospectively evaluated 110 co-infected patients, with $57 \% \mathrm{HBeAg}$ positive. Among those treated with LAM monotherapy ( $\mathrm{HBeAg}$ positive $\mathrm{N}=24$; $\mathrm{HBeAg}$ negative $\mathrm{N}=18$ ), $22 \%$ of those who were $\mathrm{HBeAg}$ positive $(\mathrm{N}=5)$ exhibited seroconversion to $\mathrm{HBeAg}$ negative over a 5-year period, which compared to 1 -year studies was no different (21\% HBeAg seroconversion). This was attributed to the development of LAM resistance over time. ${ }^{24}$

\section{Preexisting anti-HBV resistance}

The appearance of certain mutations in the HBV DNA genome is indicative of LAM resistance for those taking the medication. In the LAM trial by Lai et al, mutations to the YMDD locus that convey drug resistance were only seen in the treatment group. ${ }^{7}$ There have been studies aiming to assess pretreatment HBV genotype sequences for comparison between those that develop resistance during treatment, and those that do not. One such study analyzed pretreatment serum from 59 patients, all of which developed undetectable HBV DNA with LAM monotherapy. HBV DNA reappeared in 28 cases (47\%). Comparison of HBV genomes revealed two statistically significant mutations within the DNA encoding HBV polymerase. ${ }^{25}$ Other studies have shown mutations in the HBV genome encoding components of HBsAg, most commonly in the pre-S1 and pre-S2 regions, though the clinical significance of this is still unclear, particularly as there is overlap between the polymerase and surface antigen genes. ${ }^{26}$

It has been discovered that YMDD mutations also occur in patients who are $\mathrm{HBV}$ treatment naïve, with prevalence 
as high as $15 \%$ in one Western Chinese population. ${ }^{27} \mathrm{~A}$ multicenter review in the People's Republic of China found YMDD variants in $23 \%$ of a sample of 1,042 patients, ${ }^{28}$ and a literature review of Chinese and English publications revealed a pooled incidence of "natural" YMDD mutation of $12.21 \%$, with the People's Republic of China having the greatest incidence at $13.38 \%$, with the mean of the remaining seven countries $9.9 \% .{ }^{29}$ Studies assessing whether this leads to a decreased response to LAM are fewer in number, and the detection rate can vary depending on the assay method used. One study found that "natural"YMDD mutations disappeared following 9 months of treatment with LAM monotherapy, however the sample size was only four patients, and 9 months of treatment is a relatively short duration. ${ }^{30}$

The presence of "natural" resistance is most likely the result of spontaneous mutations that occur during virus replication. It is unclear exactly how much pre-existing resistance affects response rates to LAM. ${ }^{31}$ In practice, these patients would likely be treated with presumed LAM resistance, so long-term data on LAM monotherapy is not available.

More recent studies from Europe using deep sequencing techniques on the HBV genome in treatment-naïve patients have also confirmed the presence of low genetic barrier, primary drug-resistance mutations. ${ }^{32}$ These techniques are able to detect mutations that standard direct PCR sequencing assays cannot pick up, due to their relative lack of frequency (as low as $0.6 \%$ in one study). ${ }^{33}$ Multiple studies have shown the presence of low genetic barrier mutations that, if amplified, could convey primary drug resistance in viruses from treatment-naïve patients. ${ }^{32-34}$ Although it is still unclear what the clinical significance of these findings is, the potential for drug resistance in treatment naïve patients, coupled with the inability to detect these changes by PCR, may change the standard diagnostic test used for virus genetic profiling prior to beginning medications.

\section{LAM monotherapy may not be preferred in certain subpopulations}

Treatment recommendations for special patient populations including those with cirrhosis, liver transplantation, and those undergoing chemotherapy or other immunosuppressive treatments, are outlined in the European Association for the Study of the Liver (EASL) practice guidelines. ${ }^{35}$

Given the risk of decompensation and hepatic failure with virologic breakthrough, LAM is not recommended for these populations, since LAM loses efficacy over time with increasing rates of resistance. Long-term therapy is needed to maintain viral suppression and prevent progression of liver disease in these individuals, and the current first-line agents are ETV and TDF. A key concept in treating cirrhotics with CHB is that antiviral therapy may not prevent development of HCC, so routine monitoring with serum alpha fetoprotein (AFP) and ultrasound imaging of the liver is required. With advanced disease, achieving an undetectable HBV DNA level prior to liver transplantation will decrease the risk of HBV recurrence. ${ }^{36}$ The immunosuppression required to prevent graft rejection can also lead to reactivation and/or acute exacerbation of disease, making effective long-term chemoprophylaxis crucial in these patients.

Following liver transplantation, risk of HBV recurrence is $80 \%-100 \%$ with an associated 2-year mortality of $50 \%$ without treatment. Hepatitis B immunoglobulin (HBIG) has been used to prevent "reinfection" with HBV following liver transplantation since the early 1990s. High doses (10,000 IU) are used, with daily infusions during the first week posttransplant. Studies have been unable to standardize dosing beyond this period, but lifelong, regular infusions are currently recommended. Oral antiviral therapy is used concurrently, and the combination of LAM with HBIG reduces post-transplant HBV recurrence to as low as $0 \%-11 \%{ }^{35,37}$ There is active research addressing the question of whether a combination of oral antiviral medications can replace the need for HBIG.

Immunosuppressive treatment can lead to reactivation of $\mathrm{CHB}$, even in patients with negative $\mathrm{HBsAg}$, positive $\mathrm{HBsAb}$, and positive hepatitis B total core antibody (anti-HBc). EASL guidelines recommend that these patients should be treated with antiviral medication regardless of HBV DNA levels during the entire course of immunosuppression, and continued for at least 12 months after cessation of the immunosuppressive agents. ${ }^{35}$ LAM is currently an accepted form of treatment, as most studies were conducted using LAM, but some reviews suggest using a higher potency agent such as ETV or TDF for patients who will be immunosuppressed for longer than 6 months due to the increasing risk of LAM resistance over time. ${ }^{38}$

\section{Reducing risk of LAM anti-HBV resistance Taking a thorough history and knowing the risk factors prior to prescribing LAM treatment}

The decision to start antiviral therapy in CHB is complex, and influenced by many factors. Given that no antiviral treatment fully eradicates $\mathrm{HBV}$, knowing the resistance profile of each medication is important when choosing a regimen. A thorough 
history should be obtained to quantify all risk factors. These include the patient's age (to determine potential duration of treatment), access to health care including coverage for prescriptions, laboratory testing, and clinic visits, and ability to comply with medications. Baseline HBV DNA, HBV genotype, and HIV status should be checked. Treatment response will need to be monitored with HBV DNA levels at regular intervals, especially during the first 24 weeks. ${ }^{3}$

An accurate history of any prior treatment with LAM is crucial in determining the likelihood of resistance and the chance of response. Given that the rate of resistance increases with treatment duration, the length of prior treatment courses should be evaluated. As discussed before, 65\%-80\% of patients who have taken LAM for 5 years develop resistance. Any prior non-compliance with LAM should also be documented, and the patient should be made aware of the possibility of a poor response to treatment.

\section{When to test for LAM resistance at baseline}

Testing for resistance at baseline can help predict response to LAM, and therefore determine who is most appropriate for treatment. ${ }^{18}$ This may be especially useful in those with a prior treatment history, as their likelihood of LAM resistance is significantly higher. However, some genetic studies suggest that LAM resistance is not conveyed by specific point mutations alone. It is possible that other regions of the $\mathrm{HBV}$ genome are required to take advantage of mutations before a virus can become a resistant strain. One study in particular recommended sequencing of the entire HBV genome rather than looking for specific mutations, which may not be cost effective or easily analyzed. ${ }^{31}$ Currently, none of the guidelines recommend routine anti-HBV resistance testing in the treatment naïve population prior to starting HBV treatment.

\section{Appropriate use of LAM for HBV treatment}

The indications for treatment of HBV are discussed in the AASLD practice guidelines, and apply regardless of the type of medication chosen. LAM is no longer first-line therapy in developed countries, including the United States. The preferred agents are those with less risk of resistance, such as ETV and TDF. While HIV co-infected patients were once preferentially treated with LAM because of its dual efficacy, LAM monotherapy is now avoided if there are other resources available. This includes combination therapy with LAM and ADV or Emtricitabine-TDF. LAM monotherapy remains an option for patients with clinical indications for treatment of HBV where LAM is the only agent available, long-term access to medication is available and medical compliance is assured.

\section{Pre-treatment patient education and close clinical follow-up}

Studies assessing the benefit of patient education on compliance have seen mixed results in the amount of improvement in patient response. However, there is some evidence that patient education helps, at least short-term, ${ }^{39}$ and there is literature to support the doctor-patient relationship and effective communication as useful tools in enhancing medication adherence. ${ }^{40}$ Pre-treatment patient education can be conducted during a clinic visit, is low cost, can present essential information to patients in an easy to understand manner, and allows patients to express their concerns. Patients should fully understand the risks of the medication, the benefits to treatment, and the risks of non-compliance before starting therapy.

During treatment with LAM monotherapy, the patient should have HBV DNA levels and ALT checked at regular intervals. HBV DNA should ideally become undetectable by week 24 . Failure to respond to treatment increases the risk of resistance significantly. However, treatment should not be terminated without an alternative regimen available, as studies have shown that LAM still has efficacy, albeit significantly reduced, in the setting resistance. ${ }^{3}$

\section{Know the presentation of LAM-resistance}

This will be discussed in detail in the next section. The initial presentation of LAM resistance is usually virological breakthrough, with a rebound increase in HBV DNA levels. This can be followed by biochemical breakthrough, with elevation of ALT. More severe viral breakthrough can present as acute flare, decompensation of preexisting cirrhosis, and even fulminant hepatic failure. ${ }^{3}$

\section{De novo LAM and ADV combination treatment}

Given the superior resistance profile, good safety profile and once daily dosing regimen, ETV or TDF are now the preferred first-line agents for treatment of CHB. In certain clinical settings, including LAM failure and post-liver transplant status, combination therapy has been found to improve long-term efficacy of viral suppression. ${ }^{41}$ Although no large trials have been completed yet, the combination of ADV and LAM, given de novo, has compared favorably to LAM 
monotherapy and even been shown to be as good as ETV in achieving virological and biochemical response. ${ }^{41,42}$ Given that the largest study involved only 71 patients (31 LAM plus ADV, 40 ETV) and treatment duration was only 48 weeks, larger studies over longer periods of time are needed.

\section{Available rescue therapies for LAM anti-HBV resistance}

A successful rescue therapy for LAM resistance relies on knowledge of potential cross-resistance among HBV NAs. As summarized in Table 1, resistance to LAM conveys cross-resistance to $\mathrm{LdT}$ and ETV. ${ }^{10} \mathrm{LdT}$ and LAM share many of the same resistance mutations, so there is strong cross-resistance between the two medications and LdT is not recommended for rescue therapy by any of the guidelines. LAM resistance also lowers the resistance barrier to ETV, to the degree that even add-on ETV is not recommended, though additional mutations are still needed after LAM resistance develops. The lack of data showing cross resistance to TDF is a significant reason why it is one of the first-line rescue therapy regimens. ${ }^{3,14,35}$

When LAM resistance develops, the treatment regimen should be altered to try and prevent complications, such as virological breakthrough, biochemical breakthrough, acute hepatitis B flare, and progression of liver disease. Multiple rescue therapies have been studied over the years, as summarized in Table 2. The AASLD 2009 practice guidelines update for management of CHB recommends either a combination of LAM and ADV, or a switch to TDF in the setting of LAM resistant infection. ${ }^{3}$ The EASL practice guidelines stated that switching from LAM to TDF is the first-line rescue therapy for LAM resistance, with the addition of ADV to LAM as a second line option in case TDF is not available. ${ }^{35}$

\section{Switch to adefovir}

ADV was the first antiviral agent to be studied as rescue therapy in the setting of LAM resistance, both in "add-on" and "switch-to" strategies. Randomized controlled trials have been conducted comparing add-on ADV with placebo (continuing LAM in both groups), ${ }^{43-45}$ with results consistently showing improved virologic response in the ADV group compared to the placebo group, decline in HBV DNA in a significant number of patients, though HBeAg seroconversion was similar in both groups.

ADV resistance can become a problem with long-term monotherapy. One study separated $42 \mathrm{HBeAg}$ negative patients into treatment groups with add-on ADV therapy $(n=28)$ and switch-to ADV therapy $(n=14)$. The rate of virological and biochemical response, defined as undetectable HBV DNA and ALT levels, respectively, was not statistically significant between the two groups (90\% of add-on and $71 \%$ of switch-to patients). Response was maintained throughout

Table 2 Summary of rescue therapies in LAM resistant CHB

\begin{tabular}{|c|c|c|c|}
\hline $\begin{array}{l}\text { Rescue therapy } \\
\text { regimen }\end{array}$ & Efficacy & Concerns & Clinical application \\
\hline $\begin{array}{l}\text { Switch to ADV } \\
\text { monotherapy }\end{array}$ & $\begin{array}{l}\text { Able to achieve virologic and biochemical } \\
\text { response in a significant number of LAM } \\
\text { resistant patients }{ }^{43-45}\end{array}$ & $\begin{array}{l}\text { ADV resistance with virologic } \\
\text { breakthrough occurred in }>20 \% \\
\text { in multiple studies }{ }^{46}\end{array}$ & Not recommended \\
\hline $\begin{array}{l}\text { Switch to ETV } \\
\text { monotherapy }\end{array}$ & $\begin{array}{l}\text { Better than ADV monotherapy in reduction } \\
\text { of HBV DNA and normalization of } A L T^{8,46-48}\end{array}$ & $\begin{array}{l}\text { Virologic breakthrough due to } \\
\text { development of ETV resistance } \\
\text { (8\%-10\%); } 52,53 \text { not recommended } \\
\text { by treatment guidelines }{ }^{3,35}\end{array}$ & Not recommended \\
\hline 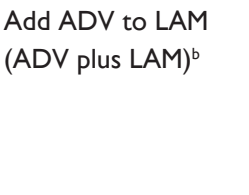 & $\begin{array}{l}\text { Similar to or greater efficacy compared to ETV } \\
\text { monotherapy; significantly less multidrug resistance } \\
\text { and virologic breakthrough compared to ADV and ETV } \\
\text { monotherapy; recommended by the AASLD and EASL } \\
\text { for LAM-resistant CHB (second line by the latter) }\end{array}$ & $\begin{array}{l}\text { Requires access to and long-term } \\
\text { compliance with two medications }\end{array}$ & $\begin{array}{l}\text { Recommended, if } \\
\text { TDF not available }\end{array}$ \\
\hline $\begin{array}{l}\text { Switch to ADV and } \\
\text { ETV (ADV plus ETV) }\end{array}$ & $\begin{array}{l}\text { Highest rates of virologic and biochemical } \\
\text { response with almost no resistance }{ }^{54,55}\end{array}$ & $\begin{array}{l}\text { Fewest number of patients tested; } \\
\text { expensive }\end{array}$ & $\begin{array}{l}\text { High cost, may not } \\
\text { be practical }\end{array}$ \\
\hline $\begin{array}{l}\text { Switch to TDF } \\
\text { monotherapy }\end{array}$ & $\begin{array}{l}\text { Switching to TDF from ADV in LAM resistant } \\
\text { CHB led to further improvement in HBV DNA } \\
\text { reduction; '0,50-52 recommended by the AASLD and } \\
\text { EASL for LAM-resistant CHB (first line by the latter); } \\
\text { similar price to ADV } 3,35\end{array}$ & $\begin{array}{l}\text { Newest medication, may not be } \\
\text { as readily accessible globally; ADV } \\
\text { resistance reduces efficacy }{ }^{57}\end{array}$ & Highly recommended \\
\hline
\end{tabular}

Notes: "aswitch" implies stopping Lamivudine; " "add on" implies continuing Lamivudine.

Abbreviations: AASLD, American Association for the Study of Liver Diseases; ADV, Adefovir; ALT, alanine aminotransferase; CHB, chronic hepatitis B; EASL, European Association for the Study of the Liver; ETV, Entecavir; HBV, hepatitis B virus; LAM, Lamivudine; TDF, Tenofovir. 
the treatment duration (median 40 months) in the add-on group, but $21 \%$ of the switch-to group developed virologic and biochemical breakthrough with evidence of ADV resistant mutations. ${ }^{46}$

The above results were corroborated in a retrospective study, which collected data from 338 patients over a 5-year period from a single center in the People's Republic of China. All had developed LAM resistance following monotherapy, and were given any one of four rescue treatments: switch-to ADV ( $\mathrm{n}=207)$, add-on ADV ( $\mathrm{n}=72)$, switch-to ETV $(n=50)$, and switch to combination ADV and ETV $(n=9)$. The incidence of drug resistance was highest in the switch-to ADV group (24\%) compared to switch-to ETV (18\%), add-on ADV (7\%), and switch-to ADV plus ETV (0\%). It was difficult to compare between groups given the significant differences in treatment groups, and the ADV plus ETV group was likely underpowered. However, it supports a preference towards add-on ADV therapy versus both switch-to ADV and switch-to ETV therapy. ${ }^{47}$ As ADV is not a preferred monotherapy agent, switching to ADV is not recommended by most guidelines.

\section{Switch to entecavir}

Switching LAM to ETV monotherapy carries a significant risk of cross-resistance to ETV, similar to the risk of ADV resistance with switch therapy. Although ETV has a superior resistance and efficacy profile when used as monotherapy in treating NA-naïve patients with $\mathrm{CHB},{ }^{46}$ and studies show higher rates of undetectable HBV DNA and normalization of ALT, even after 48 weeks, when compared to continuing LAM monotherapy. ${ }^{8,48-50}$ In one study, nearly $8 \%$ of patients with LAM anti-HBV resistant CHB developed ETV resistance after switching to ETV monotherapy for 2 years. ${ }^{50}$ A second study performed resistance monitoring on patients taking ETV over 5 years for CHB. The study population included 187 patients who had documented LAM resistance. Cumulative probability of developing genotypic ETV resistance was $6 \%, 15 \%, 36 \%, 47 \%$, and $51 \%$ in years 1 through 5 , respectively, with corresponding probabilities of virologic breakthrough of $1 \%, 11 \%, 27 \%, 41 \%$, and $43 \% .{ }^{10}$ Therefore, ETV monotherapy is not a recommended treatment option for LAM anti-HBV resistant CHB.

\section{Addition of adefovir to LAM}

There have been many randomized, controlled trials comparing the addition of ADV to LAM with ADV monotherapy and ETV monotherapy in anti-HBV resistant $\mathrm{CHB}$. As discussed above, comparisons of switch-to ADV and add-on ADV showed higher rates of virological and biochemical breakthrough among the latter group, as well as lower rates of virological breakthrough and development of ADV resistance. This is the reason why add-on ADV is preferred over ADV monotherapy for LAM anti-HBV resistant $\mathrm{CHB}{ }^{46,47}$

Multiple studies have shown that add-on ADV therapy has a higher rate of virological and biochemical response compared to switch-to ETV therapy, including Zhao et al, where the add-on ADV group showed a statistically significant $(P<0.001)$ improvement in HBV DNA reduction and normalization of ALT compared to the switch-to ADV group after 12 months of treatment. ${ }^{47}$ Comparisons of results in the add-on ADV group with those in the switch-to ETV group showed similar degrees of reduction of HBV DNA at 6 months of rescue therapy, but add-on ADV became more effective after 12 months compared to switch-to ETV.

Another retrospective study reviewed 104 patients with genotypic LAM resistance and both virological and biochemical breakthrough who underwent rescue therapy with either switch to ETV $(n=24)$, switch to ADV $(n=44)$, or ADV add-on $(n=36)$. After 6 months of rescue therapy, there was already a statistically significant difference in achievement of undetectable HBV DNA in the ADV add-on group (68.6\%) compared to the switch-to ETV group $(33.3 \%, P=0.003)$. However, at 12 months, this difference was no longer significant as both ADV add-on and switch-to ETV groups began to show decreasing HBV DNA levels. Switch-to ADV therapy was inferior to both. Rates of normalization of ALT were similar, and not statistically significant. $^{51}$

Although some randomized controlled trials directly comparing ADV add-on to switch-to ETV showed no statistically significant difference in rates of virological and biochemical response, ${ }^{52}$ the development of ETV cross-resistance separated the two treatment regimens. A meta-analysis of 3 randomized controlled trials and 3 cohort studies corroborated this data, with no statistical differences in virological response, normalization of ALT and $\mathrm{HBeAg}$ seroconversion at 48 weeks of treatment. The rate of virological breakthrough was higher in the ETV group (relative risk $=0.16, P<0.0001) .{ }^{53}$

\section{Switch to adefovir and entecavir combination therapy}

Switching therapy to a combination of ADV and ETV showed excellent virological and biochemical response rates with little development of resistant virus strains. One study randomized 
91 patients with documented LAM resistant mutations into treatment groups using ADV monotherapy $(n=29)$, ADV add-on $(\mathrm{n}=30)$, and ADV plus ETV combination therapy $(n=32)$. Treatment duration was 24 months. Mean reduction in HBV DNA in units of $\log _{10} \mathrm{IU} / \mathrm{L}$ was $-3.78,-4.92$, and -5.58 for the switch-to ADV, ADV plus LAM, and ADV plus ETV groups after 24 months of therapy, respectively $(P<0.05)$. Achievement of undetectable HBV DNA was only statistically significant at the 6 month point, with ADV plus ETV having the highest rate at that point (78\% compared to $27.5 \%$ of ADV monotherapy and $57 \%$ of ADV plus LAM patients). Normalization of ALT was not statistically significant at either 6 or 12 month follow-up. Virological breakthrough occurred in 8/29 (27.6\%) patients switched to ADV monotherapy, 4/30 (13.3\%) patients who had ADV added to LAM, and $0 / 32$ patients in the ADV plus ETV group $(P<0.05) .{ }^{54}$

ADV plus ETV regimen has also been shown to be superior to ADV plus LAM in direct comparison studies. For example, in a single center randomized active-control trial from Korea, 90 patients with lamivudine-resistant $\mathrm{CHB}$ with HBV DNA $>2000 \mathrm{IU} / \mathrm{mL}$ after at least 24 weeks of ADV plus LAM treatment were randomized into combination therapy with ETV plus ADV $(n=45)$ or continuation of ADV plus LAM $(\mathrm{n}=45)$ and treated for 52 weeks. Undetectable HBV DNA levels were achieved in 13/45 (29\%) of ETV plus ADV patients compared to 2/45 (4\%) of ADV plus LAM patients $(P=0.004)$. Mean reduction of HBV DNA in $\log _{10} \mathrm{IU} / \mathrm{mL}$ from baseline was also significantly better in the ETV plus ADV group ( -2.2 versus -0.6 in the ADV plus LAM group, $P<0.001)$. No genotypic mutations causing resistance to ETV or ADV were detected in the ETV plus ADV group. ${ }^{53}$

\section{Switch to tenofovir monotherapy}

TDF is a nucleoside analog structurally similar to ADV. It was approved for treatment of CHB in 2008. Randomized, controlled trials demonstrated superior efficacy of TDF compared to ADV at reducing HBV DNA levels and normalizing ALT, including the landmark phase 3 study that included $286 \mathrm{HBeAg}$-positive patients and $375 \mathrm{HBeAg}-$ negative patients treated for 48 weeks. Successful viral suppression, defined as HBV DNA $<400$ copies/mL, was more successful in the TDF treated groups compared to the ADV treated groups of both patient populations (76\% versus $13 \%$ with $P<0.01$ among HBeAg-positive patients, 93\% versus $63 \%$ with $P<0.01$ among HBeAg-negative patients). ${ }^{56}$ Based on these and other similar results, TDF became the new first-line agent alongside ETV for nucleoside naïve patients with $\mathrm{CHB} .{ }^{3,35}$

Data for primary therapy was followed by smaller studies evaluating TDF monotherapy as rescue therapy for LAM resistance, particularly LAM resistance that did not respond to ADV-based rescue regimens. Most of the earlier research involved patients with HIV co-infection. A larger, retrospective cohort study was conducted in 2010 with 131 HBV infected patients who had poor response to either LAM monotherapy, ADV monotherapy, or ADV plus LAM combination therapy. Seventy of those patients (62\%) had LAM-resistant mutations and all were treated with TDF monotherapy for 1-year. Mean baseline HBV DNA was 8.3 (range 4.1-9.7) $\log _{10}$ copies/mL, and following treatment mean HBV DNA was 3 (range 2.6-4.2). The probability of achieving HBV DNA $<400$ copies/mL was $100 \%$ with TDF monotherapy, and $90 \%$ of patients without ADV resistance achieved this after 1 year. ${ }^{57}$

Another study, which was not randomized, treated 35 patients with LAM-resistant CHB with TDF and 18 patients with ADV. Among the TDF group included 21 patients with HIV co-infection, and five patients who were status post-kidney transplant and on immunosuppression. Mean reduction of HBV DNA in the TDF group after 48 weeks of treatment was $-5.6 \log _{10}$ copies $/ \mathrm{mL}$ compared to $-2.5 \log _{10}$ copies/mL in the ADV group $(P<0.001)$. There was a statistically significant difference in the rate of normalization of ALT after 48 weeks (85\% TDF versus $57 \%$ ADV, $P=0.008)$. LAM was continued for 1 -year in the HIV co-infected group; all other patients stopped LAM at the beginning of treatment with TDF or ADV. ${ }^{58}$ A third study retrospectively reviewed 106 patients with LAM-resistant CHB on rescue therapy using either ADV monotherapy or ADV plus LAM combination therapy. These patients had been defined as suboptimal responders to ADV based on a persistently detectable HBV DNA after 6 months of ADV treatment. Eighteen were switched to TDF monotherapy, and these patients demonstrated a statistically significant increase in the rate of maintaining virological response (undetectable HBV DNA) at 1 -year follow-up (87.5\% with TDF, $8.7 \%$ with ADV, $P<0.001){ }^{59}$

The only limiting factor to response to TDF was resistance to ADV, which reduced probability of achieving $\mathrm{HBV}$ DNA $<400$ copies $/ \mathrm{mL}$ from $100 \%$ to $52 \%(P<0.001) .{ }^{59}$ Patients with prior exposure to ADV and LAM-resistant CHB requiring rescue therapy may need to be tested for resistance to ADV prior to beginning TDF. Cost may be an issue, though the price of TDF is comparable to that of ADV. 


\section{Summary}

Although using LAM to treat CHB carries the risk of resistance, the medication is still widely used throughout the world due to its relatively low cost and high availability. LAM resistance can lead to virological and biochemical breakthrough in patients on this treatment, leading to progression of liver disease. There are many factors that can affect a patient's risk for developing LAM resistance. Understanding these is critically important, and all of the precautions discussed above should be exercised to reduce this risk, if LAM must be used as monotherapy for CHB. Deciding on rescue therapy in the setting of LAM resistance can be challenging, due to the risk of multidrug resistance. At this time, adding ADV to LAM or switching to TDF monotherapy are the first-line recommendations for rescue therapy. Patients who have received ADV before may not respond due to ADV resistance. Switching LAM to ADV and ETV combination therapy also seems to be effective, but is more costly and therefore not as strongly recommended.

\section{Disclosure}

Dr Ke-Qin Hu received grants from Bristol-Myers Squibb Company, Gilead Sciences Inc, Genentech Pharmaceutics, Merck Corporation, and Vertex Pharmaceuticals. Dr Hu is also a member of the speaker bureau of Bristol-Myers Squibb Company, Genentech Pharmaceutics, Gilead Sciences Inc, Merck Corporation, and Vertex Pharmaceuticals. The authors have no other conflicts of interest to declare.

\section{References}

1. cdc.gov [homepage on the Internet]. Hepatitis B frequently asked questions for the public. Centers for Disease Control; 2008 [updated June 9, 2009; cited January 1, 2013]. Available from: http://www.cdc. gov/hepatitis/b/bFAQ.htm\#statistics. Accessed January 1, 2013.

2. http://www.who.int/en/ [homepage on the Internet]. Hepatitis B fact sheet. World Health Organization; 2012 [cited January 1, 2013]. Available from: http://www.who.int/mediacentre/factsheets/fs204/en/. Accessed January 1, 2013.

3. Lok AS, McMahon BJ. American Association for the Study of Liver Diseases (AASLD) Practice Guidelines for Management of Chronic Hepatitis B: Update 2009. Hepatology. 2009. Available from: http://www. aasld.org/practiceguidelines/documents/bookmarked $\% 20$ practice $\% 20$ guidelines/chronic_hep_b_update_2009\%208_24_2009.pdf. Accessed January 1, 2013.

4. Schütte K, Bornschein J, Malfertheiner P. Hepatocellular carcinoma epidemiological trends and risk factors. Dig Dis. 2009;27(2):80-92.

5. Wong DK, Cheung AM, O'Rourke K, Naylor CD, Detsky AS, Heathcote J. Effect of alpha-interferon treatment in patients with hepatitis $\mathrm{B}$ e antigen-positive chronic hepatitis B. A meta-analysis. Ann Intern Med. 1993;119(4):312-323.

6. Yuen MF, Lai CL. Treatment of chronic hepatitis B: Evolution over two decades. J Gastroenterol Hepatol. 2011;26 Suppl 1:138-143.

7. Lai CL, Chien RN, Leung NW, et al. A one-year trial of lamivudine for chronic hepatitis B. Asia Hepatitis Lamivudine Study Group. $N$ Engl J Med. 1998;339(2):61-68.
8. Chang TT, Gish RG, de Man R, et al. A comparison of entecavir and lamivudine for HBeAg-positive chronic hepatitis B. N Engl J Med. 2006;354(10):1001-1010.

9. Lok AS, Lai CL, Leung N, et al. Long-term safety of lamivudine treatment in patients with chronic hepatitis B. Gastroenterology. 2003;125(6):1714-1722.

10. Tenney DJ, Rose RE, Baldick CJ, et al. Long-term monitoring shows hepatitis B virus resistance to entecavir in nucleoside-naïve patients is rare through 5 years of therapy. Hepatology. 2009;49(5):1503-1514.

11. Liaw YF, Leung N, Kao JH, et al. Asian-Pacific consensus statement on the management of chronic hepatitis B: a 2008 update. Hepatol Int. 2008;2(3):263-283.

12. Dienstag JL, Schiff ER, Wright TL, et al. Lamivudine as initial treatment for chronic hepatitis B in the United States. N Engl J Med. 1999;341(17):1256-1263.

13. Zoulim F. Hepatitis B virus resistance to antiviral drugs: where are we going? Liver Int. 2011;31 Suppl 1:111-116.

14. Fung J, Lai CL, Seto WK, Yuen MF. Nucleoside/nucleotide analogues in the treatment of chronic hepatitis B. J Antimicrob Chemother. 2011;66(12):2715-2725.

15. Yuen MF, Sablon E, Hui CK, Yuan HJ, Decraemer H, Lai CL. Factors associated with hepatitis B virus DNA breakthrough in patients receiving prolonged lamivudine therapy. Hepatology. 2001;34(4 Pt 1): 785-791.

16. Fournier C, Zoulim F. Antiviral therapy of chronic hepatitis B: prevention of drug resistance. Clin Liver Dis. 2007;11(4):869-892, ix.

17. Wang C, Fan R, Sun J, Hou J. Prevention and management of drug resistant hepatitis B virus infections. J Gastroenterol Hepatol. 2012;27(9):1432-1440.

18. Marcellin P, Sung J, Piratvisuth T. Avoiding and managing lamivudine resistance in chronic hepatitis B: current approaches and potential strategies including pegylated interferon. Liver Int. 2010;30(5): $657-668$.

19. Lai CL, Gane E, Liaw YF, et al. Telbivudine versus lamivudine in patients with chronic hepatitis B. N Engl J Med. 2007;357(25):2576-2588.

20. Gane EJ. The Roadmap concept: using early on-treatment virologic responses to optimize long-term outcomes for patients with chronic hepatitis B. Hepatol Int. 2008;2(3):304-307.

21. Hsieh TH, Tseng TC, Liu CJ, et al. Hepatitis B virus genotype B has an earlier emergence of lamivudine resistance than genotype C. Antivir Ther (Lond). 2009;14(8):1157-1163.

22. Mello FC, Fernandes CA, Gomes Sde A. Antiviral therapy against chronic hepatitis B in Brazil: high rates of lamivudine resistance mutations and correlation with HBV genotypes. Mem Inst Oswaldo Cruz. 2012;107(3):317-325.

23. Benhamou $Y$, Bochet M, Thibault V, et al. Long-term incidence of hepatitis $\mathrm{B}$ virus resistance to lamivudine in human immunodeficiency virus-infected patients. Hepatology. 1999;30(5):1302-1306.

24. Kosi L, Reiberger T, Payer BA, et al. Five-year on-treatment efficacy of lamivudine-, tenofovir- and tenofovir + emtricitabine-based HAART in HBV-HIV-coinfected patients. J Viral Hepat. 2012;19(11):801-810.

25. Sueki R, Maekawa S, Miura M, et al. Correlation between pretreatment viral sequences and the emergence of lamivudine resistance in hepatitis B virus infection. J Med Virol. 2012;84(9):1360-1368.

26. Chen $\mathrm{CH}$, Lee CM, Tung WC, et al. Evolution of full-length HBV sequences in chronic hepatitis B patients with sequential lamivudine and adefovir dipivoxil resistance. J Hepatol. 2010;52(4):478-485.

27. Zhao J, Guo Y, Yan Z, Liang P, Zhang J, Liu Y. The natural YMDD mutations of hepatitis B virus in Western China. Scand J Infect Dis. 2012;44(1):44-47.

28. Tan YW, Ge GH, Zhao W, et al. YMDD motif mutations in chronic hepatitis B antiviral treatment naïve patients: a multi-center study. Braz J Infect Dis. 2012;16(3):250-255.

29. Tan Y, Ding K, Su J, et al. The naturally occurring YMDD mutation among patients chronically infected HBV and untreated with lamivudine: a systematic review and meta-analysis. PLOS ONE. 2012;7(3):e32789. 
30. Lee SH, Kim HS, Byun IS, et al. Pre-existing YMDD mutants in treatment-naïve patients with chronic hepatitis B are not selected during lamivudine therapy. J Med Virol. 2012;84(2):217-222.

31. Thai H, Campo DS, Lara J, et al. Convergence and coevolution of hepatitis B virus drug resistance. Nat Commun. 2012;3:789.

32. Svicher V, Cento V, Salpini R, et al. Role of hepatitis B virus genetic barrier in drug-resistance and immune-escape development. Dig Liver Dis. 2011;43(12):975-983.

33. Margeridon-Thermet S, Shulman NS, Ahmed A, et al. Ultra-deep pyrosequencing of hepatitis B virus quasispecies from nucleoside and nucleotide reverse-transcriptase inhibitor (NRTI)-treated patients and NRTI-naive patients. J Infect Dis. 2009;199(9):1275-1285.

34. Solmone M, Vincenti D, Prosperi MC, Bruselles A, Ippolito G, Capobianchi MR. Use of massively parallel ultradeep pyrosequencing to characterize the genetic diversity of hepatitis B virus in drug-resistant and drug-naive patients and to detect minor variants in reverse transcriptase and hepatitis B S antigen. J Virol. 2009;83(4):1718-1726.

35. European Association for the Study of the Liver. EASL clinical practice guidelines: Management of chronic hepatitis B virus infection. J Hepatol. 2012;57(1):167-185.

36. Papatheodoridis GV, Cholongitas E, Archimandritis AJ, Burroughs AK. Current management of hepatitis B virus infection before and after liver transplantation. Liver Int. 2009;29(9):1294-1305.

37. Buchanan C, Tran TT. Current status of liver transplantation for hepatitis B virus. Clin Liver Dis. 2011;15(4):753-764.

38. Rapti IN, Hadziyannis SJ. Treatment of special populations with chronic hepatitis B infection. Expert Rev Gastroenterol Hepatol. 2011;5(3):323-339.

39. Haynes RB, Ackloo E, Sahota N, McDonald HP, Yao X. Interventions for enhancing medication adherence [review]. Cochrane Database Syst Rev. 2008;2:CD000011.

40. Bonner JE, Barritt AS, Fried MW, Evon DM. Tangible resources for preparing patients for antiviral therapy for chronic hepatitis C. Dig Dis Sci. 2012;57(6):1439-1444.

41. Fan XH, Geng JZ, Wang LF, et al. De novo combination therapy with lamivudine and adefovir dipivoxil in chronic hepatitis B patients. World J Gastroenterol. 2011;17(43):4804-4809.

42. Wang LC, Chen EQ, Cao J, et al. De novo combination of lamivudine and adefovir versus entecavir monotherapy for the treatment of naïve HBeAg-negative chronic hepatitis B patients. Hepatol Int. 2011;5(2): 671-676.

43. Perrillo R, Hann HW, Mutimer D, et al. Adefovir dipivoxil added to ongoing lamivudine in chronic hepatitis B with YMDD mutant hepatitis B virus. Gastroenterology. 2004;126(1):81-90.

44. Perrillo RP, Hann HW, Schiff E, et al. Extended treatment with lamivudine and adefovir dipivoxil in chronic hepatitis B patients with lamivudine resistance. Hepatol Int. 2011;5(2):654-663.

45. Sung JJ, Lai JY, Zeuzem S, et al. Lamivudine compared with lamivudine and adefovir dipivoxil for the treatment of $\mathrm{HBeAg-positive} \mathrm{chronic}$ hepatitis B. J Hepatol. 2008;48(5):728-735.

46. Rapti I, Dimou E, Mitsoula P, Hadziyannis SJ. Adding-on versus switching-to adefovir therapy in lamivudine-resistant $\mathrm{HBe} A g-n e g a t i v e$ chronic hepatitis B. Hepatology. 2007;45(2):307-313.

47. Zhao P, Wang C, Huang L, Xu D, Li T. Comparison of rescue strategies in lamivudine-resistant patients with chronic hepatitis B. Antiviral Res. 2012;96(2):100-104.

48. Lai CL, Shouval D, Lok AS, et al. Entecavir versus lamivudine for patients with HBeAg-negative chronic hepatitis B. $N$ Engl J Med. 2006;354(10):1011-1020.
49. Sherman M, Yurdaydin C, Sollano J, et al. Entecavir for treatment of lamivudine-refractory, HBeAg-positive chronic hepatitis B. Gastroenterology. 2006;130(7):2039-2049.

50. Sherman M, Yurdaydin C, Simsek H, et al. Entecavir therapy for lamivudine-refractory chronic hepatitis B: improved virologic, biochemical, and serology outcomes through 96 weeks. Hepatology. 2008;48(1):99-108.

51. Kim HJ, Park JH, Park DI, et al. Rescue therapy for lamivudine-resistant chronic hepatitis B: comparison between entecavir $1.0 \mathrm{mg}$ monotherapy, adefovir monotherapy and adefovir add-on lamivudine combination therapy. J Gastroenterol Hepatol. 2010;25(8):1374-1380.

52. Ryu HJ, Lee JM, Ahn SH, et al. Efficacy of adefovir add-on lamivudine rescue therapy compared with switching to entecavir monotherapy in patients with lamivudine-resistant chronic hepatitis B. J Med Virol. 2010;82(11):1835-1842.

53. Sheng YJ, Liu JY, Tong SW, et al. Lamivudine plus adefovir combination therapy versus entecavir monotherapy for lamivudine-resistant chronic hepatitis B: a systematic review and meta-analysis. Virol $J$. 2011;8:393.

54. Ha M, Zhang G, Diao S, et al. Rescue therapy for lamivudine-resistant chronic hepatitis B: adefovir monotherapy, adefovir plus lamivudine or entecavir combination therapy. Intern Med. 2012;51(12):1509-1515.

55. Lim YS, Lee JY, Lee D, et al. Randomized trial of entecavir plus adefovir in patients with lamivudine-resistant chronic hepatitis B who show suboptimal response to lamivudine plus adefovir. Antimicrob Agents Chemother. 2012;56(6):2941-2947.

56. Marcellin P, Heathcote EJ, Buti M, et al. Tenofovir disoproxil fumarate versus adefovir dipivoxil for chronic hepatitis B. $N$ Engl J Med. 2008;359(23):2442-2455

57. van Bömmel F, de Man RA, Wedemeyer H, et al. Long-term efficacy of tenofovir monotherapy for hepatitis B virus-monoinfected patients after failure of nucleoside/nucleotide analogues. Hepatology. 2010;51(1):73-80.

58. van Bömmel F, Wünsche T, Mauss S, et al. Comparison of adefovir and tenofovir in the treatment of lamivudine-resistant hepatitis B virus infection. Hepatology. 2004;40(6):1421-1425.

59. Ong A, Wong VW, Wong GL, Chan HY, Tse CH, Chan HL. Management options for lamivudine-resistant chronic hepatitis B patients with suboptimal virological suppression by adefovir. Aliment Pharmacol Ther. 2011;34(8):972-981.

60. Allen MI, Deslauriers M, Andrews CW, et al. Identification and characterization of mutations in hepatitis B virus resistant to lamivudine. Lamivudine Clinical Investigation Group. Hepatology. 1998;27(6): 1670-1677.

61. Tenney DJ, Levine SM, Rose RE, et al. Clinical emergence of entecavir-resistant hepatitis $\mathrm{B}$ virus requires additional substitutions in virus already resistant to Lamivudine. Antimicrob Agents Chemother. 2004;48(9):3498-3507.

62. Lai CL, Leung N, Teo EK, et al. A 1-year trial of telbivudine, lamivudine, and the combination in patients with hepatitis B e antigen-positive chronic hepatitis B. Gastroenterology. 2005;129(2):528-536.

63. Locarnini S, Hatzakis A, Heathcote J, et al. Management of antiviral resistance in patients with chronic hepatitis B. Antivir Ther (Lond). 2004;9(5):679-693. 


\section{Publish your work in this journal}

Drug Design, Development and Therapy is an international, peerreviewed open-access journal that spans the spectrum of drug design and development through to clinical applications. Clinical outcomes, patient safety, and programs for the development and effective, safe, and sustained use of medicines are a feature of the journal, which

has also been accepted for indexing on PubMed Central. The manuscript management system is completely online and includes a very quick and fair peer-review system, which is all easy to use. Visit http://www.dovepress.com/testimonials.php to read real quotes from published authors.

Submit your manuscript here: http://www.dovepress.com/drug-design-development-and-therapy-journal 